\title{
Perioperative infections in congenital heart disease
}

\author{
Indah K. Murni, ${ }^{1}$ Graeme MacLaren, ${ }^{2,3}$ Debra Morrow, ${ }^{4}$ Parvathi Iyer, ${ }^{5}$ Trevor Duke ${ }^{3,6}$ \\ ${ }^{1}$ Department of Pediatrics, Dr Sardjito Hospital, Faculty of Medicine Universitas Gadjah Mada, Yogyakarta, Indonesia; \\ ${ }^{2}$ Cardiothoracic ICU, National University Health System, Singapore; ${ }^{3}$ Intensive Care Unit, Royal Children's Hospital, \\ Melbourne, Australia; ${ }^{4}$ Department of Nursing, Cardiovascular Intensive Care Unit, Boston Children's Hospital, Boston, \\ Massachusetts, United States of America; ${ }^{5}$ Department of Pediatric and Congenital Heart Surgery, Fortis Escorts Heart Institute, \\ New Delhi, India; ${ }^{6}$ Centre for International Child Health, University of Melbourne and MCRI, Melbourne, Australia
}

\begin{abstract}
Background: Perioperative infections have significant consequences for children with congenital heart disease (CHD), which can manifest as acute or chronic infection followed by poor growth and progressive cardiac failure. The consequences include delayed or higher-risk surgery, and increased postoperative morbidity and mortality. Methods: A systematic search for studies evaluating the burden and interventions to reduce perioperative infections in children with CHD was undertaken using PubMed. Results: Limited studies conducted in low- to middle-income countries demonstrated the large burden of perioperative infections among children with CHD. Most studies focussed on infections after surgery. Few studies evaluated strategies to prevent preoperative infection or the impact of infection on decision-making around the timing of surgery. Children with CHD have multiple risk factors for infections including delayed presentation, inadequate treatment of cardiac failure, and poor nutrition. Conclusions: The burden of perioperative infections is high among children with CHD, and studies evaluating the effectiveness of interventions to reduce these infections are lacking. As good nutrition, early corrective surgery, and measures to reduce nosocomial infection are likely to play a role, practical steps can be taken to make surgery safer.
\end{abstract}

Keywords: Perioperative infection; children; CHD; low- and middle-income country

Received: 15 September 2017; Accepted: 15 September 2017

$\mathrm{C}$ HD IS A MAJOR CONTRIBUTOR TO THE GLOBAL burden of childhood illness and death, with an approximate incidence of $0.8 \%$ of all live births. Half of all deaths among patients with $\mathrm{CHD}$ occur during the first year of life, especially in countries where access to surgery is limited. In lowincome countries, limitations of human resources and facilities for cardiac surgery and postoperative management mean that, for many children, correctable congenital heart lesions are not addressed or, if they are, the perioperative period is more hazardous because of complications related to delayed surgery.

Correspondence to: I. K. Murni, MD, PhD, Department of Pediatrics, Dr Sardjito Hospital, Faculty of Medicine Universitas Gadjah Mada, Jalan Kesehatan No 1, Sekip, Yogyakarta, Indonesia, 55281. Tel:+62 816 685894; E-mail: indah. kartika.m@ugm.ac.id
Children with CHD have increased risk and severity of infection. Impaired nutrition and pulmonary congestion predisposes to pneumonia and other lower respiratory tract infections. Furthermore, some forms of CHD are associated with genetic abnormalities or syndromes that lead to some level of immunosuppression or increased susceptibility to infection. ${ }^{1}$

This review focusses on aspects of perioperative infection in children with CHD, particularly the frequency of these infections, diagnostic issues, impact on timing of surgery and outcomes, and interventions to reduce perioperative infections.

\section{Methods \\ Search strategy}

A systematic search of the English-language literature was undertaken using PubMed with the following 
terms: ((Infect* OR "Infection"[Mesh] OR infection OR "perioperative infection" OR "preoperative infection" OR "postoperative infection" OR "intraoperative infection" OR sepsis OR septicemia OR endotoxemia) AND (cardiac OR "congenital heart disease") AND (child* OR pediatric OR pediatrics OR paediatric OR paediatrics OR infant OR neonat*)).

All studies that evaluated the epidemiology of perioperative infections - preoperative, intraoperative, and postoperative infection - in children with $\mathrm{CHD}$, the impact of infections on health outcomes, and interventions to reduce perioperative infections were identified. Studies with duplicated data were excluded.

\section{Data extraction and outcome measures}

After reading the full texts, the eligibility of all studies identified using the inclusion criteria outlined above were independently assessed. Using a standardised data-extraction form, we summarised study details including authors, year of publication, and country or countries where the study was performed; study design; population and number of subjects; outcomes and results including timing of infection (preoperative, intraoperative, and postoperative), type and definition of infections - community- or hospital-acquired infections including bloodstream infection or sepsis, pneumonia, viral infections, infective endocarditis, other and definition used for diagnostic criteria - frequency of infections - prevalence, cumulative incidence, incidence density type of organism(s), and other - risk factors, predictors, and impact of infections.

Types of infection were grouped as follows: preoperative infections including community-acquired infections and hospital-acquired infections or nosocomial infections; intraoperative infections; and postoperative infections including nosocomial bloodstream infection or catheter-associated bloodstream infection or sepsis, ventilator-associated pneumonia, urinary tract infection or catheterassociated urinary tract infection, and surgical site infection or wound infection.

Outcomes were as follows: frequency and risk factors for perioperative infections; diagnosis of perioperative infections; impact of infection on timing of surgery; impact of infection on outcomes; and intervention(s) to reduce perioperative infections. The frequency of perioperative infections according to two standard metrics was expressed as episodes/ 1000 patient-days or cases/100 patients - the percentage of patients who developed a perioperative infection. Impact of infections on health outcomes was assessed based on associated mortality and morbidity.

\section{Results}

The search identified 16,172 articles, of which 62 satisfied the inclusion criteria (Fig 1). Among eligible published studies, 41 were conducted in high-income countries (Supplementary material, Table 1) and 21 in low- or middle-income countries (Supplementary material, Table 2).

\section{Epidemiology of perioperative infections in high-income countries}

All studies were conducted in tertiary urban or teaching hospitals. Among studies conducted in highincome settings, 15 were in European countries, ${ }^{2-16}$ 19 in the United States of America, ${ }^{17-35}$ one in Australia, ${ }^{36}$ three elsewhere in North America, ${ }^{37-39}$ and three in Asia. ${ }^{40-42}$ There were three nonrandomised interventional studies, $, 22,2332$ cohort studies, $^{2-5,7-20,24,27-29,31-34,36-38,40-42}$ three casecontrol studies, ${ }^{21,25,30}$ and three cross-sectional studies. $^{26,35,39}$ The majority of studies were conducted on children, three studies focussed specifically on neonates, ${ }^{18,27,37}$ and three studies involved both children and adults. $3,4,42$

\section{Preoperative infections}

There were 10 studies describing preoperative infections. These included acute respiratory tract infections - upper respiratory tract infection,

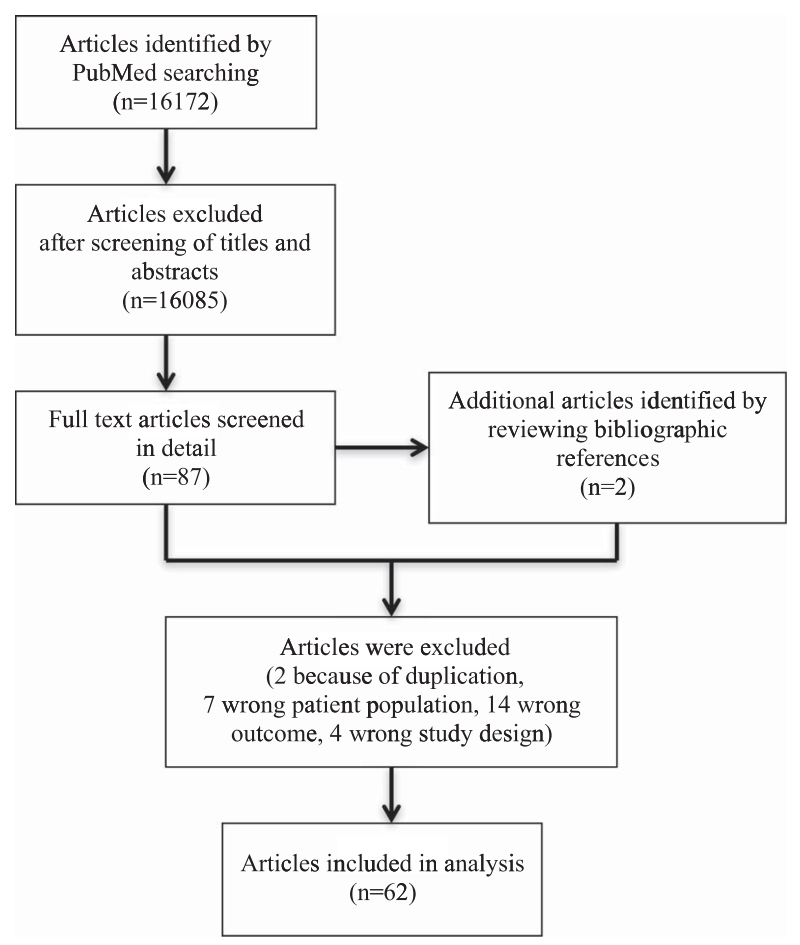

Figure 1.

Search strategy for articles. 
Table 1. Practical considerations in preventing, identifying, and treating perioperative infections in children with cardiac disease, focussed particularly on low- and middle-income settings.

(1) Optimise nutrition before surgery, including protein-energy intake and micronutrients

(2) Treat all children preoperatively with an antihelminth drug; in malaria endemic regions obtain a blood film and treat accordingly; obtain a Mantoux test and screen for TB and HIV as indicated

(3) Ensure optimal haemoglobin and treat with iron and haematinics in the weeks before surgery

(4) Ensure clean and intact skin and treat skin infection or scabies before surgery

(5) Chlorhexidine body wash preoperatively reduces Gram-positive bacterial infection

(6) Consider delaying surgery for 2-4 weeks in the setting of acute respiratory viral infection

(7) Do not use prophylactic antibiotics except a single dose at the time of induction of anaesthesia

(8) No biomarker is specific for infection in the postoperative period, and most biomarkers are elevated in the days after cardiopulmonary bypass

(9) The diagnosis of bacterial sepsis after cardiopulmonary bypass must be based on the combination of clinical and laboratory information useful thresholds include the immature/total granulocyte ratio ( $>0.2$ or rising after 2 days postoperatively), procalcitonin level $>2 \mathrm{ng} / \mathrm{ml}$, or rising after 2 days postoperatively

(10) Always take a blood culture and other relevant cultures before commencing antibiotics, and be prepared to scale down or cease after 48 hours if cultures are negative

(11) Hand hygiene is vital - always adhere to the 5 moments of hand hygiene (World Health Organization)

(12) Study the antibiogram of pathogens in your unit, so antibiotics can be properly targeted in the setting of sepsis

bronchiolitis, pneumonia, and respiratory syncytial virus infections $s^{5,10,11,20,40}-$ sepsis, $^{18}$ and infective endocarditis. ${ }^{4,24,39,41}$

Among children with cardiac disease, the cumulative incidence of preoperative hospitalisation from respiratory syncytial virus (RSV) infection was $0.5 \%(2518 / 549,265)$ to $9.6 \%(58 / 602)$ in children $<2$ years of age with mortality of $1.9 \%$ of 8127 to $4.9 \%$ of 549,265 patients. ${ }^{5,11,20,40}$ Risk factors for hospital admission due to acute respiratory tract infections were malnutrition, infant, male, chromosomal abnormalities, wheezing, inadequate respiratory syncytial virus prophylaxis, and sibling $<11$ years of age. ${ }^{10}$ In children with haemodynamically significant CHD, those who underwent surgery early might be less often hospitalised compared with those who underwent surgery later. ${ }^{11}$

In the United States of America, the cumulative incidence of preoperative sepsis in children with CHD was $5.6 \%(656 / 11,638) .{ }^{18}$ The most common bacteria isolated in children with sepsis and CHDs were Gram-positive organisms (64\%), including coagulasenegative Staphylococcus and Staphylococcus aureus. ${ }^{18}$ Risk factors for sepsis included young age, high complexity of cardiac surgery, history of cardiothoracic surgery, preoperative length of hospital stay $>24$ hours, preoperative ventilator use, genetic syndromes, ${ }^{19}$ length of hospital stay $>10$ days, and cyanotic CHD. ${ }^{15}$

Incidence density of preoperative infective endocarditis among children with CHD was $0.32 / 1000$ to $6.1 / 1000$ hospitalisations. ${ }^{39,41}$ In a study involving children and adults, the cumulative incidence of infective endocarditis in CHD was as high as $5.5 \%$ $(45 / 821){ }^{4}$ Community-associated pathogens in children with infective endocarditis included viridans streptococci (38\%) and Staphylococcus aureus (19\%), whereas healthcare-associated infective endocarditis pathogens were Candida spp (27\%), coagulasenegative staphylococci (20\%), and Gram-negative bacilli $(16 \%) .{ }^{41}$ Risk factors for preoperative infective endocarditis were cyanotic CHD, atrioventricular septal defects, left-sided lesions, and age $<30$ years.

\section{Postoperative infections}

Many studies have described postoperative infections in children undergoing cardiac surgery. Among those studies, the most common infection studied was surgical site infection with varied incidence of $1.2 \%$ (12/981) to $48 \%$ (22/46). . $6,17,23,25-27,31-33,35,37,38,42^{2}$ Risk factors for surgical site infections were duration of surgery, ${ }^{17,21}$ age $<1$ month, ${ }^{17,27,32}$ blood transfusion, ${ }_{32}$ parenteral nutrition, ${ }^{32}$ perioperative medical device, ${ }^{32}$ excessive bleeding within 24 hours after surgery, and incorrect timing of preoperative antibiotics. ${ }^{27}$

Postoperative bacteraemia occurred in $1.5 \%(15 /$ 992) to $10.2 \%$ (13/127). ${ }^{8,19,27,28,37}$ The most common isolated bacteria in infants with healthcare-associated bloodstream infections included Staphylococcus epidermidis, Escherichia coli, Enterobacter cloacae, Bacillus spp, Staphylococcus aureus, Enterococcus spp, and Klebsiella spp. ${ }^{37}$ Fungal infection or invasive candidaemia post-cardiac surgery occurred in $0.4 \%(6 / 1540)$ to $5.9 \%$ (10/ 169). ${ }^{3,7,16}$ Risk factors for postoperative bloodstream infections were use of central venous catheters, ${ }^{27,30}$ blood transfusion, use of steroids, and antibiotic prophylaxis for more than 2 days after surgery. ${ }^{33}$

\section{Pulmonary infections}

RSV infection was described in 3\% of 1737 children after cardiac surgery. ${ }^{36}$ The incidence of nosocomial pneumonia in infants undergoing cardiac surgery was $6.2 \%(8 / 127)^{37}$ and incidence density of ventilatorassociated pneumonia in children was $17.1 / 1000$ 
patient-days. ${ }^{12}$ Haemophilus influenzae, Moraxella catarrhalis, Staphylococcus aureus, and Pseudomonas aeruginosa were the most common pathogens isolated in children with ventilator-associated pneumonia. Risk factors for ventilator-associated pneumonia were PRISM III score $>10$ and transfusion of fresh-frozen plasma. ${ }^{12}$

\section{Epidemiology of perioperative infections in low- and middle-income countries}

Among 21 studies in low- and middle-income countries, four were conducted in China, ${ }^{43-46}$ four in India, ${ }^{47-50}$ three in Brazil, ${ }^{51-53}$ two in Saudi Arabia $^{54,55}$, and one each in Argentina, ${ }^{56}$ Bosnia Herzegovina, ${ }^{57}$ Iran, ${ }^{58}$ Ethiopia, ${ }^{59}$ Guatemala, ${ }^{60}$ and Sudan. ${ }^{61}$ One multicentre study involving 17 low- to middle-income countries included India, Pakistan, Colombia, Russia, China, Dominican Republic, Brazil, Argentina, El Salvador, Ukraine, Peru, Mexico, Belarus, Vietnam, Uganda, Guatemala, and Bangladesh. ${ }^{62}$

There were five different study designs including one randomised controlled trial, ${ }^{53}$ one non-randomised interventional before-after study, ${ }^{62} 16$ cohort studies, ${ }^{43-45,47-50,52,54-57,59,60,63}$ one case-control study, ${ }^{51}$ and three cross-sectional studies. ${ }^{46,58,61}$ Most studies were conducted in children, with two in neonates ${ }^{47,57}$ and two involving children and adult patients. $^{43,50}$

\section{Preoperative infection}

There were seven studies evaluating preoperative infections among children with CHD in low- to middle-income country settings. In India, the incidence of preoperative infection was $33 \%(28 / 84){ }^{48}$ In Brazil, the incidence of sepsis was $4.6 \%$ of 127 patients $^{52}$ and late sepsis was $18 \%(14 / 80)$ in Bosnia Herzegovina. ${ }^{57}$ The incidence of pneumonia before surgery was $16.5 \%$ among 127 children with CHD in Brazil. ${ }^{52}$ The most common bacteria in infants with preoperative infections included Staphylococcus aureus, Klebsiella spp, and Serratia spp.

In China, the incidence of infective endocarditis related to CHD was 79\% (27/34) and infective endocarditis related to rheumatic heart disease was $6 \%(2 / 34) .{ }^{45}$ Bacteria was isolated from children with preoperative infective endocarditis including Staphylococcus aureus (58\%), Strepcococcus viridians (8\%), Enterococcus faecium (8\%), other Gram-positive cocci $(17 \%)$, and other Gram-negative bacilli $(3 \%){ }^{45}$ Among children and adults with culture-positive infective endocarditis, viridans group streptococci (56\%), Staphylococcus spp (15\%), Pseudomonas spp (7\%), Corynebacterium dipbtheriae (4\%), Acinetobacter baumannii (4\%), Salmonella enterica serovar Typhi (4\%), and Granulicatella adiacens (4\%) were isolated. ${ }^{50}$ In Sudan, 160 children with CHD might develop rates of dental caries and gingivitis as high as 4 and $2 \%$, respectively. ${ }^{61}$

\section{Postoperative infection}

Among studies evaluating postoperative infections, the prevalence of infection was varied ranging from $7.8 \%$ of 203 children in Iran to $40 \%$ of 300 children in China. ${ }^{46,58}$ Microbes isolated from children with postoperative infection were $66.6 \%$ Gram-positive and $26.7 \%$ Gram-negative bacteria, and $6.7 \%$ were fungi. The most common pathogens were Staphylococcus epidermidis, Staphylococcus aureus, Enterococcus spp, Pseudomonas aeruginosa, and Candida albicans. ${ }^{46}$ Risk factors for postoperative infections were length of hospital stay, use of combined antibiotics, use of hormone therapy, mechanical ventilation, and indwelling central venous catheter. ${ }^{46}$

The incidence of postoperative sepsis or bloodstream infection across 17 countries was $7 \%$ of 15,049 in children, and 21\% (17/330) in neonates, after cardiac surgery. ${ }^{47,49,54,56,62}$ The most common pathogens isolated were Gram-negative bacteria in $67 \%$, Pseudomonas aeruginosa in $28 \%$, and Enterobacter spp in $22 \%$ of children with postoperative bloodstream infection. ${ }^{54}$ Risk factors for bloodstream infections after cardiac surgery were malnutrition, high complexity of surgery, delayed sternal closure, prolonged central venous catheter use, and prolonged ICU and hospital stay. ${ }^{54}$ Candidaemia occurred in $0.07 \%$ children. ${ }^{51}$ Risk adjustment for congenital heart surgery (RACHS)-1 score $\geqslant 3$, use of acid suppression, and thrombocytopaenia were independent risk factors for candidaemia. ${ }^{51}$

The incidence of surgical site infection was $2.5 \%$ of 350 children with CHD in Argentina to $12.7 \%$ (55/330) in India, ${ }^{47,49,56}$ with Gram-positive cocci being the most frequent pathogens isolated. ${ }^{56}$ The incidence of nosocomial pneumonia approached $21 \%$ (67/331) in China to $44 \%$ of 6864 children in India ${ }^{44,49,53}$ and ventilator-associated pneumonia was $6.2 \%(105 / 1688)$ in China to $18.3 \%$ of 160 children in Brazil. ${ }^{43,53,55}$ The most common pathogens isolated in children with ventilatorassociated pneumonia were Gram-negative bacilli, followed by Gram-positive cocci and fungi. ${ }^{43,44,55}$ Risk factors for ventilator-associated pneumonia included prolonged cardiopulmonary bypass, use of parenteral nutrition, ICU length of stay, ${ }^{55}$ mechanical ventilation, extubation failure, emergency surgery, and blood transfusion. ${ }^{43}$

Around $7 \%$ children developed catheter-associated urinary tract infections, ${ }^{49,63}$ with Gram-negative bacteria most commonly isolated. Duration of foley catheter, congenital anomalies of the kidney and 
urinary tract, and genetic syndromes were independent risk factors for catheter-associated urinary tract infections. ${ }^{63}$

\section{Impact of infection on timing of surgery (global)}

There were no studies evaluating the impact of perioperative infections on timing of cardiac surgery. We could only find studies that evaluated the impact of timing surgery on later occurrence of infections. Early surgery was associated with less frequent hospitalisations owing to respiratory syncytial virus infections in children with haemodynamically significant CHD. ${ }^{11}$ Older postnatal age at surgery might increase preoperative infections in infants born small for gestational age compared with non-small gestational age. ${ }^{34}$

\section{Impact of infections on health outcomes (global)}

Several published studies in high-income countries evaluated the impact of infection on outcomes including mortality and other morbidity or complications related to infections. Studies on preoperative and postoperative infection conducted in highincome countries revealed that those infections might be associated with increased mortality and length of hospital stay. Hospitalisation related to preoperative respiratory syncytial virus infections was associated with mortality rates of $1.9 \%$ of 549,265 in Taiwan to $4.9 \%$ of 549,265 in the United States of America. ${ }^{20,40}$ Children with preoperative infective endocarditis related to $\mathrm{CHD}$ had mortality rates of $2.8 \%$ of 3840 children in the United States of America to $12 \%(6 / 51)$ in Israel. ${ }^{24,41}$ A study in Spain involving children and adolescents with infective endocarditis demonstrated mortality rates of $24 \%$ of 821 children with CHD. ${ }^{4}$

Children with postoperative infections had increased mortality, prolonged length of stay, and increased healthcare costs. Nosocomial infection increased healthcare costs and length of stay. ${ }^{29}$ Postoperative viral infection was associated with prolonged paediatric ICU stay and prolonged intubation. ${ }^{36}$ Postoperative major infections including bacteraemia, mediastinitis, and endocarditis were associated with increased mortality and length of hospital stay. ${ }^{8}$ In a study conducted in Korea, sternal wound infections were associated with mortality rates as high as $18.2 \%(28 / 154) .{ }^{42}$ The adverse impact of surgical site infection on length of stay, prolonged mechanical ventilation, and need for vasoactive agents were also demonstrated. ${ }^{31}$

In low- to middle-income countries, studies evaluating the impact of perioperative infections on health outcomes are limited. Preoperative infection in children with CHD may be associated with a fourfold increase in mortality. ${ }^{48}$ Ventilator-associated pneumonia was associated with mortality rates of $11 \%$ of 137 children in Saudi Arabia to $25.7 \%$ $(27 / 105)$ in China. ${ }^{43,55}$ A multicentre study conducted in 17 low- to middle-income countries demonstrated an association between postoperative major infection and mortality of $6.3 \%$ of 15,049 children. $^{62}$

\section{Interventions to reduce perioperative infection and their effectiveness (global)}

There were three intervention studies conducted in high-income countries to reduce postoperative infections including catheter-associated bloodstream infection and surgical site infection. A quality improvement study using interrupted time series design implemented a multifaceted intervention to reduce catheter-associated bloodstream infection. These included a central line-associated bloodstream infection prevention initiative including establishment of a unit-based infection control, nurse position, education, real-time feedback on central line-associated bloodstream infection data, implementation of central venous line bundles, and introduction of daily goal sheets on rounds. After implementing these measures, central line-associated bloodstream infection rates significantly decreased from 7.8 infections per 1000 catheter-days to 2.3 infections per 1000 catheter-days. ${ }^{22}$ A before-andafter study implemented an interdisciplinary infection control programme including preoperative, intraoperative, and postoperative measures to reduce surgical site infection. After implementing the intervention, the incidence of surgical site infection decreased significantly from $10.9 \%$ (95\% confidence interval (CI) $4.7-18.8$ ) to $1.92 \%$ (95\% CI $0.4-5.52$ ) in the preand post-intervention period, respectively. ${ }^{6}$ Another before-and-after study implemented measures to reduce surgical site infection using chlorhexidine in the operative and postoperative periods, standardised intravenous antibiotic within 5-60 minutes of the initial surgical incision, with initiation of cardiopulmonary bypass, and every 3 hours in the intraoperative period, antibiotics for 48 hours, and dressing removal at 48 hours in the postoperative period. These measures were associated with a reduction of sternal wound infection rates from 3.8 to $2.1 \%{ }^{23}$

There were two intervention studies conducted in low-to middle-income countries. The first study was a randomised controlled trial aimed to reduce the risk of ventilator-associated pneumonia. The patients were randomised to receive oral hygiene with $0.12 \%$ chlorhexidine gluconate or placebo preoperatively and twice a day postoperatively until paediatric ICU discharge or death. After implementing the intervention, the incidence of nosocomial pneumonia was 29.8 versus $24.6 \%$ 
$(p=0.46)$ and the incidence of ventilator-associated pneumonia was 18.3 versus $15 \%(p=0.57)$ in the chlorhexidine and the control group, respectively. ${ }^{53}$

Another study conducted in 17 low- to middleincome countries used a before-and-after study design. An intervention was implemented using webinars that targeted three key drivers: safe perioperative practices, infection reduction, and team-based practice and audited registry data. After implementing the intervention, the risk-adjusted inhospital mortality was significantly lower in both 2011 (standardised mortality ratio $0.71,95 \% \mathrm{CI}$ $0.62-0.81$ ) and 2012 (standardised mortality ratio $0.76,95 \%$ CI $0.69-0.83$ ) relative to $2010 .{ }^{62}$

\section{Discussion of perioperative infections in low- and middle-income countries}

The limited studies of children with CHD in low- and middle-income countries demonstrate that perioperative infections are common. Many risk factors exist for infection in children with CHD and some children have multiple risk factors that may accrue if there is delayed presentation, inadequate treatment, or poor nutrition.

Poor nutrition is associated with adverse outcomes in children undergoing cardiac surgery, including infection. ${ }^{64}$ There is a complex interaction between heart disease, malnutrition, immunity and infection, and adverse outcomes or complications. During starvation, cardiac myocytes may atrophy. Children suffering from severe malnutrition may exhibit cardiovascular abnormalities including hypotension, cardiac arrhythmias, cardiomyopathy, or cardiac failure. $^{65}$ Malnutrition impairs many aspects of immune function, including mucosal barrier function, microbiocidal activity of granulocytes, reduced T-cell function, and reduced complement activity. ${ }^{66}$ Micronutrient deficiencies have a myriad of effects on immunity and cardiac function: severe iron deficiency anaemia can lead to high-output cardiac failure or reduced left ventricular contractility; thiamine deficiency causes vasodilatation and high-output cardiac failure; and selenium deficiency weakens myocyte function. Electrolyte imbalances are more common in children with severe malnutrition and cardiac disease and have many effects on the heart. Severe hypophosphataemia, for example, weakens heart muscle and reduces stroke volume, and hypomagnesaemia increases the risk of arrhythmias. Pulmonary congestion increases susceptibility to pneumonia and other lower respiratory tract infections in children with left to right shunts, which in turn increases exposure to antibiotics and increases the likelihood of antibiotic-resistant bacterial colonisation. This puts the child at an increased risk of serious invasive bacterial sepsis during and after surgery. Several gene polymorphisms associated with CHD have been linked with an increased susceptibility to sepsis, and several malformation syndromes or chromosomal syndromes, including DiGeorge syndrome and CHARGE syndrome, link CHD with immunodeficiency. Chronic hypoxia associated with cyanotic CHD may affect immune responses. Children with CHD frequently are exposed to invasive devices, which increase the risk of nosocomial infection. Ischaemia-reperfusion injury following cardiopulmonary bypass frequently results in a state of reduced functional immune response, which also increases the risk of nosocomial infections. ${ }^{1}$

The impact of these diverse risk factors on perioperative mortality and morbidity in CHD in developing countries is uncertain, but they need to be taken into account in surgical planning. Children with CHD need an individualised risk assessment while awaiting surgery, and this involves an assessment of infection risk, nutritional intake, growth and micronutrients, and adequacy of control of cardiac failure.

\section{Bacterial infection epidemiology}

While Gram-positive infections were still the most common cause of postoperative sepsis in high-income countries, Gram-negative sepsis was prevalent in children with postoperative bloodstream infections in low- to middle-income countries. Gram-negative sepsis is associated with significant morbidity, higher mortality, and development of antibiotic resistance.

About one-third of children with CHD developed preoperative infection in low- to middle-income countries. We could find no study evaluating the impact of perioperative infections on timing of surgery in low- or middle-income settings, although early surgery was associated with less frequent hospitalisations owing to acute infections in children with haemodynamically significant CHD. Whether the risk of cardiac surgery in children with preoperative infections can be mitigated in any way if surgery has to proceed is still uncertain.

\section{Implications for patient care and further research}

Formulating effective screening and prevention strategies to reduce infections is important. This requires early detection of perioperative infections, prompt management, adequate postoperative care, and early nutritional support. There is considerable scope for further research, in particular focussing on the influence of infection on the timing of surgery, as well as specific infections, which have received insufficient attention in this patient population, including 
preoperative Gram-negative bacteraemia, human immunodeficiency virus, and tuberculosis.

We propose 12 practical rules to reduce perioperative infections among those children in low- and middle-income countries (Table 1).

\section{Acknowledgements}

None.

\section{Financial Support}

This review received no specific grant from any funding agency, commercial, or not-for-profit sectors.

\section{Conflicts of Interest}

None.

\section{Supplementary material}

To view supplementary material for this article, please visit https://doi.org/10.1017/S1047951117002578

\section{References}

1. Wheeler DS, Jeffries HE, Zimmerman JJ, Wong HR, Carcillo JA Sepsis in the pediatric cardiac intensive care unit. World J Pediatr Congenit Heart Surg 2011; 2: 393-399.

2. Algra SO, Driessen MM, Schadenberg AW, et al. Bedside prediction rule for infections after pediatric cardiac surgery. Intensive Care Med 2012; 38: 474-481.

3. Forstner $\mathrm{C}$, Lassnigg A, Tobudic $\mathrm{S}$, et al. A prospective analysis of invasive candidiasis following cardiac surgery: severity markers are predictive. J Infect 2013; 66: 528-535.

4. Fortun J, Centella T, Martin-Davila P, et al. Infective endocarditis in congenital heart disease: a frequent community-acquired complication. Infection 2013; 41: 167-174.

5. Granbom E, Fernlund E, Sunnegardh J, Lundell B, Naumburg E. Respiratory tract infection and risk of hospitalization in children with congenital heart defects during season and off-season: a Swedish national study. Pediatr Cardiol 2016; 37: 1098-1105.

6. Izquierdo-Blasco J, Campins-Marti M, Soler-Palacin P, et al. Impact of the implementation of an interdisciplinary infection control program to prevent surgical wound infection in pediatric heart surgery. Eur J Pediatr 2015; 174: 957-963.

7. Jaworski R, Haponiuk I, Irga-Jaworska N, et al. Fungal infections in children in the early postoperative period after cardiac surgery for congenital heart disease: a single-centre experience. Interact Cardiovasc Thorac Surg 2016; 23: 431-437.

8. Kansy A, Jacobs JP, Pastuszko A, et al. Major infection after pediatric cardiac surgery: external validation of risk estimation model. Ann Thorac Surg 2012; 94: 2091-2095.

9. Lomtadze M, Chkhaidze M, Mgeladze E, Metreveli I, Tsintsadze A. Incidence and risk factors of nosocomial infections after cardiac surgery in Georgian population with congenital heart diseases. Georgian Med News 2010; 1: 7-10.

10. Medrano Lopez C, Garcia-Guereta L. Community-acquired respiratory infections in young children with congenital heart diseases in the palivizumab era: the Spanish 4-season civic epidemiologic study. Pediatr Infect Dis J 2010; 29: 1077-1082.

11. Resch B, Kurath-Koller S, Hahn J, Raith W, Kostenberger M, Gamillscheg A. Respiratory syncytial virus-associated hospitalizations over three consecutive seasons in children with congenital heart disease. Eur J Clin Microbiol Infect Dis 2016; 35: 1165-1169.

12. Roeleveld PP, Guijt D, Kuijper EJ, Hazekamp MG, de Wilde RB, de Jonge E. Ventilator-associated pneumonia in children after cardiac surgery in the Netherlands. Intensive Care Med 2011; 37: 1656-1663.

13. Sarvikivi E, Lyytikainen O, Nieminen H, Sairanen H, Saxen H. Nosocomial infections after pediatric cardiac surgery. Am J Infect Control 2008; 36: 564-569.

14. Toth R, Szanto P, Prodan Z, et al. Down syndrome and postoperative complications after paediatric cardiac surgery: a propensity-matched analysis. Interact Cardiovasc Thorac Surg 2013; 17: 691-697.

15. Valera M, Scolfaro C, Cappello N, et al. Nosocomial infections in pediatric cardiac surgery, Italy. Infect Control Hosp Epidemiol 2001; 22: 771-775.

16. Wessel A, Simon C, Regensburger D. Bacterial and fungal infections after cardiac surgery in children. Eur J Pediatr 1987; 146: 31-33.

17. Allpress AL, Rosenthal GL, Goodrich KM, Lupinetti FM, Zerr DM. Risk factors for surgical site infections after pediatric cardiovascular surgery. Pediatr Infect Dis J 2004; 23: 231-234.

18. Ascher SB, Smith PB, Clark RH, et al. Sepsis in young infants with congenital heart disease. Early Hum Dev 2012; 88 (Suppl 2): S92-S97.

19. Barker GM, O'Brien SM, Welke KF, et al. Major infection after pediatric cardiac surgery: a risk estimation model. Ann Thorac Surg 2010; 89: 843-850.

20. Chu PY, Hornik CP, Li JS, Campbell MJ, Hill KD. Respiratory syncytial virus hospitalisation trends in children with haemodynamically significant heart disease, 1997-2012. Cardiol Young 2017; 27: 16-25.

21. Costello JM, Graham DA, Morrow DF, et al. Risk factors for surgical site infection after cardiac surgery in children. Ann Thorac Surg 2010; 89: 1833-1841.

22. Costello JM, Morrow DF, Graham DA, Potter-Bynoe G, Sandora TJ, Laussen PC. Systematic intervention to reduce central lineassociated bloodstream infection rates in a pediatric cardiac intensive care unit. Pediatrics 2008; 121: 915-923.

23. Delgado-Corcoran C, Van Dorn CS, Pribble C, et al. Reducing pediatric sternal wound infections: a quality improvement project. Pediatr Crit Care Med 2017; 18: 461-468.

24. Gupta S, Sakhuja A, MsGrath E, Asmar B. Trends, microbiology, and outcomes of infective endocarditis in children during 2000-2010 in the United States. Congenit Heart Dis 2016; 12: 196-201.

25. Harder EE, Gaies MG, Yu S, et al. Risk factors for surgical site infection in pediatric cardiac surgery patients undergoing delayed sternal closure. J Thorac Cardiovasc Surg 2013; 146: 326-333.

26. McNeil JC, Ligon JA, Hulten KG, et al. Staphylococcus aureus infections in children with congenital heart disease. J Pediatr Infect Dis 2013; 2: 337-344.

27. Murray MT, Krishnamurthy G, Corda R, et al. Surgical site infections and bloodstream infections in infants after cardiac surgery. J Thorac Cardiovasc Surg 2014; 148: 259-265.

28. O'Brien JE Jr, Marshall JA, Tarrants ML, Stroup RE, Lofland GK. Intraoperative hyperglycemia and postoperative bacteremia in the pediatric cardiac surgery patient. Ann Thorac Surg 2010; 89: 578-583.

29. Pasquali SK, He X, Jacobs ML, et al. Hospital variation in postoperative infection and outcome after congenital heart surgery. Ann Thorac Surg 2013; 96: 657-663.

30. Prasad PA, Dominguez TE, Zaoutis TE, et al. Risk factors for catheter-associated bloodstream infections in a pediatric cardiac intensive care unit. Pediatr Infect Dis J 2010; 29: 812-815.

31. Sochet AA, Cartron AM, Nyhan A, et al. Surgical site infection after pediatric cardiothoracic surgery. World J Pediatr Congenit Heart Surg 2017; 8: 7-12. 
32. Sohn AH, Schwartz JM, Yang KY, Jarvis WR, Guglielmo BJ, Weintrub PS. Risk factors and risk adjustment for surgical site infections in pediatric cardiothoracic surgery patients. Am J Infect Control 2010; 38: 706-710

33. Turcotte RF, Brozovich A, Corda R, et al. Health care-associated infections in children after cardiac surgery. Pediatr Cardiol 2014; 35: $1448-1455$.

34. Wei D, Azen C, Bhombal S, Hastings L, Paquette L. Congenital heart disease in low-birth-weight infants: effects of small for gestational age (SGA) status and maturity on postoperative outcomes. Pediatr Cardiol 2015; 36: 1-7.

35. Woodward CS, Son M, Calhoon J, Michalek J, Husain SA. Sternal wound infections in pediatric congenital cardiac surgery: a survey of incidence and preventative practice. Ann Thorac Surg 2011; 91: 799-804.

36. Moynihan $\mathrm{K}$, Barlow $\mathrm{A}$, Alphonso $\mathrm{N}$, et al. Impact of viral respiratory pathogens on outcomes after pediatric cardiac surgery. Pediatr Crit Care Med 2017; 18: 219-227.

37. Glass HC, Bowman C, Chau V, et al. Infection and white matter injury in infants with congenital cardiac disease. Cardiol Young 2011; 21: 562-571.

38. Pollock EM, Ford-Jones EL, Rebeyka I, et al. Early nosocomial infections in pediatric cardiovascular surgery patients. Crit Care Med 1990; 18: 378-384.

39. Rushani D, Kaufman JS, Ionescu-Ittu R, et al. Infective endocarditis in children with congenital heart disease: cumulative incidence and predictors. Circulation 2013; 128: 1412-1419.

40. Chiu SN, Shao PL, Chen HC, et al. Risk of respiratory syncytial virus infection in cyanotic congenital heart disease in a subtropical area. J Pediatr 2016; 171: 25-30.

41. Marom D, Levy I, Gutwein O, Birk E, Ashkenazi S. Healthcareassociated versus community-associated infective endocarditis in children. Pediatr Infect Dis J 2011; 30: 585-588.

42. Shin HJ, Jhang WK, Park JJ, Yun TJ. Impact of delayed sternal closure on postoperative infection or wound dehiscence in patients with congenital heart disease. Ann Thorac Surg 2011; 92: 705-709.

43. Sheng W, Xing QS, Hou WM, et al. Independent risk factors for ventilator-associated pneumonia after cardiac surgery. J Invest Surg 2014; 27: 256-261.

44. Tan L, Sun X, Zhu X, Zhang Z, Li J, Shu Q. Epidemiology of nosocomial pneumonia in infants after cardiac surgery. Chest 2004; 125: 410-417.

45. Wang W, Sun H, Lv T, Tian J. Retrospective studies on pediatric infective endocarditis over 40 years in a mid-west area of China. Cardiology 2014; 128: 88-91.

46. Zhang J, Yuan Y, Li P, et al. Postoperative nosocomial infections among children with congenital heart disease. Pak J Med Sci 2014; 30: 554-557.

47. Bakshi KD, Vaidyanathan B, Sundaram KR, et al. Determinants of early outcome after neonatal cardiac surgery in a developing country. J Thorac Cardiovasc Surg 2007; 134: 765-771.

48. Patwary ME, Khan MS, Marwah A, Singh V, Shekhawat S, Sharma R. Arterial switch for transposition of the great arteries with large ventricular septal defect and for Taussig Bing anomaly: experience from a tertiary care center in the developing world. World J Pediatr Congenit Heart Surg 2015; 6: 413-421.

49. Sahu MK, Siddharth B, Choudhury A, et al. Incidence, microbiological profile of nosocomial infections, and their antibiotic resistance patterns in a high volume cardiac surgical intensive care unit. Ann Card Anaesth 2016; 19: 281-287.
50. Senthilkumar S, Menon T, Subramanian G. Epidemiology of infective endocarditis in Chennai, South India. Indian J Med Sci 2010; 64: 187-191.

51. de Araujo Motta F, Dalla-Costa LM, Dominguez Muro M, Lenzi A, Picharski GL, Burger M. Risk adjustment for congenital heart surgery score as a risk factor for candidemia in children undergoing congenital heart defect surgery. Pediatr Infect Dis J 2016; 35: $1194-1198$.

52. Faria PF, Nicolau JA, Melek MZ, de Oliveira Nde S, Bermudez BE, Nisihara RM. Association between congenital heart defects and severe infections in children with Down syndrome. Rev Port Cardiol 2014; 33: 15-18.

53. Jacomo AD, Carmona F, Matsuno AK, Manso PH, Carlotti AP. Effect of oral hygiene with $0.12 \%$ chlorhexidine gluconate on the incidence of nosocomial pneumonia in children undergoing cardiac surgery. Infect Control Hosp Epidemiol 2011; 32: 591-596.

54. Abou Elella R, Najm HK, Balkhy H, Bullard L, Kabbani MS Impact of bloodstream infection on the outcome of children undergoing cardiac surgery. Pediatr Cardiol 2010; 31: 483-489.

55. Shaath GA, Jijeh A, Faruqui F, Bullard L, Mehmood A, Kabbani MS. Ventilator-associated pneumonia in children after cardiac surgery. Pediatr Cardiol 2014; 35: 627-631.

56. Rosanova MT, Allaria A, Santillan A, et al. Risk factors for infection after cardiovascular surgery in children in Argentina. Braz J Infect Dis 2009; 13: 414-416.

57. Hadzimuratovic E, Dinarevic SM, Hadzimuratovic A. Sepsis in premature newborns with congenital heart disease. Congenit Heart Dis 2010; 5: 435-438.

58. Mirzaei M, Mirzaei S, Sepahvand E, Koshkaki AR, Jahromi MK. Evaluation of complications of heart surgery in children with congenital heart disease at Dena Hospital of Shiraz. Glob J Health Sci 2016; 8: 33-38.

59. Moges T, Gedlu E, Isaakidis P, et al. Infective endocarditis in Ethiopian children: a hospital based review of cases in Addis Ababa. Pan Afr Med J 2015; 20: 75.

60. Vida VL, Leon-Wyss J, Larrazabal A, Cruz S, Castaneda AR. Mediastinitis in pediatric cardiac surgery: treatment and costeffectiveness in a low-income country. Pediatr Cardiol 2007; 28: 163-166.

61. Mohamed Ali H, Berggreen E, Nguyen D, et al. Dental plaque microbial profiles of children from Khartoum, Sudan, with congenital heart defects. J Oral Microbiol 2017; 9: 1281556.

62. Jenkins KJ, Castaneda AR, Cherian KM, et al. Reducing mortality and infections after congenital heart surgery in the developing world. Pediatrics 2014; 134: e1422-e1430.

63. Kabbani MS, Ismail SR, Fatima A, et al. Urinary tract infection in children after cardiac surgery: incidence, causes, risk factors and outcomes in a single-center study. J Infect Public Health 2016; 9: 600-610.

64. Mitting R, Marino L, Macrae D, Shastri N, Meyer R, Pathan N. Nutritional status and clinical outcome in postterm neonates undergoing surgery for congenital heart disease. Pediatr Crit Care Med 2015; 16: 448-452.

65. Ocal B, Unal S, Zorlu P, Tezic HT, Oguz D. Echocardiographic evaluation of cardiac functions and left ventricular mass in children with malnutrition. J Paediatr Child Health 2001; 37: 14-17.

66. Rytter MJ, Kolte L, Briend A, Friis H, Christensen VB. The immune system in children with malnutrition - a systematic review. PloS One 2014; 9: e105017. 